\title{
Energy distribution functions of ions impinging on substrate in microwave plasma
}

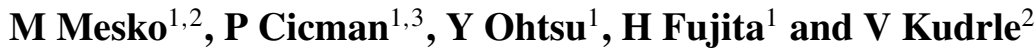 \\ ${ }^{1}$ Department of Electrical and Electronic Engineering, Saga University, Honjo-Machi 1, \\ Saga 840-8502, Japan \\ ${ }^{2}$ Department of Physical Electronics, Masaryk University, Kotlarska 2,CZ-61137 Brno, \\ Czech Republic \\ ${ }^{3}$ Institute of Ion Physics, Leopold-Franzens-University Innsbruck, Technikerstrasse 25, \\ A-6020 Innsbruck, Austria
}

E-mail: mesko@physics.muni.cz

Received 3 July 2003

Published 14 January 2004

Online at stacks.iop.org/JPhysD/37/438 (DOI: 10.1088/0022-3727/37/3/022)

\begin{abstract}
In this work, a large area microwave plasma reactor is used, in which a rf-biased substrate is exposed to argon plasma. A directional energy analyser mounted in the substrate holder was used to measure ion energy distribution functions in argon plasma. It was found that a resonance behaviour between ion transit motion and the substrate rf-bias oscillation yielded important enhancement of ion energy when the rf-bias and ion plasma frequencies reached roughly the same values.
\end{abstract}

\section{Introduction}

The energy and the flux of ions impinging on surfaces exposed to low-pressure plasma are important factors that determine the chemical structure and the physical properties of the surface layers. Ion energy distribution functions (IEDFs) have been measured in various discharges generated by planar, inductively coupled sources $[1,2,12]$, ECR sources $[3,4,11]$ and helicons $[5,6]$. These studies have investigated the dependence of ion energy on pressure $[5,8]$, source power $[2,7]$ and applied magnetic fields $[3,8,9]$. However, the dependence of IEDF on rf-bias voltage and frequency has not been investigated up to now in sufficient detail; only references $[3,5,10]$ report IEDF measured with rf-bias applied.

High-density plasma-etching equipment often employs two power supplies in order to separately adjust ion density and ion energy. The source power level controls ion density, whereas ion energies are controlled with a separate dc or rf-bias power supply connected to the substrate holder. The ion energy analyser (IEA) mounted in the substrate holder is used to measure the distribution function of energy of impinging ions.

This work presents measurements of energy distribution functions of ions impinging on the substrate in an argon microwave plasma as a function of a wide range of physically interesting rf-bias frequencies, $0.1 f_{\mathrm{pi}} \leqslant f_{\mathrm{rf}} \leqslant 10 f_{\mathrm{pi}}$, where $f_{\mathrm{pi}}$ is the ion plasma frequency and $f_{\mathrm{rf}}$ is the rf-bias frequency.

\section{Experimental apparatus}

The experiments were performed in a cylindrical shaped vacuum chamber made of stainless steel with $310 \mathrm{~mm}$ inner diameter and $300 \mathrm{~mm}$ in length. The top wall of the chamber was in fact a fused silica disc with thickness $12 \mathrm{~mm}$. Microwaves of frequency $2.45 \mathrm{GHz}$ were generated in a magnetron source and were channelled to the chamber via a waveguide. A three-stub tuner and power circulator were located between the magnetron source and the chamber.

A transition as shown in figure 1 was used for transformation of the microwave power from the rectangular waveguide to the chamber. In this case, the microwaves propagate along a metallic rod antenna made of aluminium with a length of $140 \mathrm{~mm}$ and diameter of $17 \mathrm{~mm}$. The rod antenna was placed at the centre of the fused silica plate and connected to the crossbar transition. This crossbar transition provides conversion from the dominant $\mathrm{TE}_{10}$ mode in the rectangular guide to the dominant TEM in the coaxial waveguide.

For investigation of the ion distribution functions, a substrate holder of $100 \mathrm{~mm}$ diameter was placed in the 


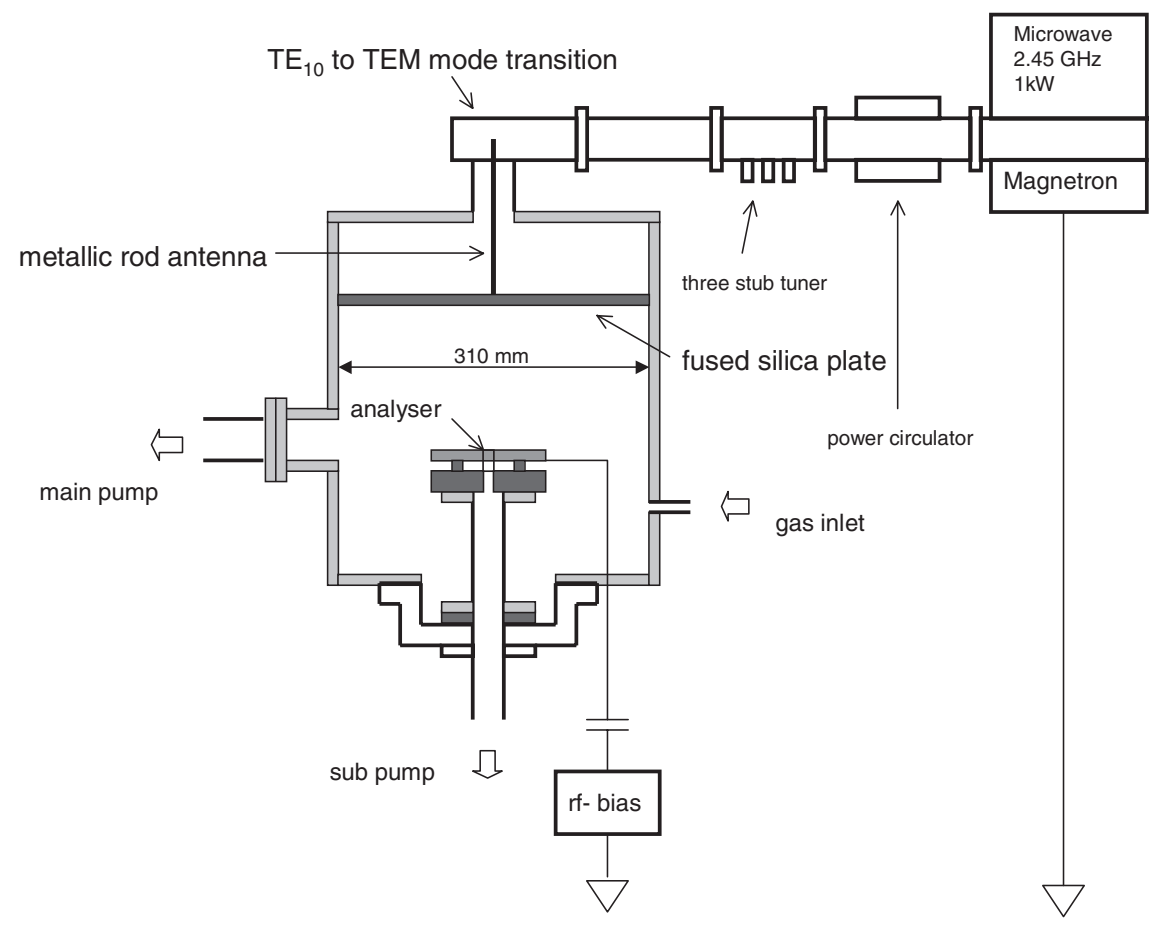

Figure 1. Experimental set-up.

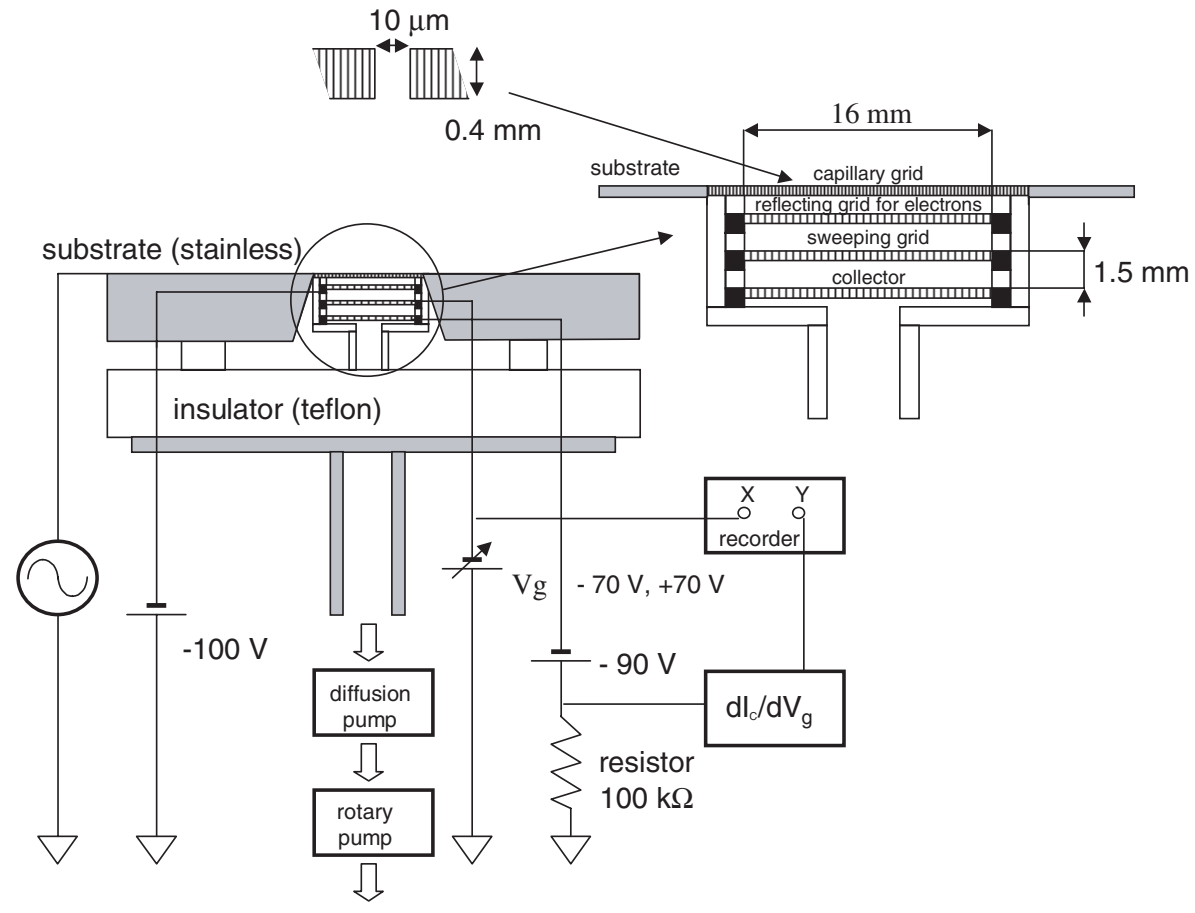

Figure 2. Schematic diagram of IEA mounted in substrate holder.

chamber, as shown in figure 1 , at a position $z=140 \mathrm{~mm}$ below the fused silica plate. In order to measure the distribution functions of ions impinging on the substrate, a gridded IEA was mounted in the centre of the substrate. The analyser consists of three grids and a collector as shown in figure 2. The front grid of the analyser was in fact a capillary plate (HAMAMATSU Photonics, J5022-11, open area ratio 55\%) with angular resolution $1-2^{\circ}[13,14]$. The second grid, third grid and the collector were made of $400 \mathrm{mesh} \mathrm{inch}^{-1}$ stainless steel grid. They were spot-welded on $0.5 \mathrm{~mm}$ thin stainless steel rings. The grid discs were separated by Teflon rings, and the housing of the analyser was made also from Teflon. The effective diameter of the analyser was $16 \mathrm{~mm}$. The capillary plate was conductively connected with the substrate holder, at which the rf-bias voltages (peak to peak 20-80 V, $200 \mathrm{kHz}-13.56 \mathrm{MHz}$ ) were applied. In order to suppress 


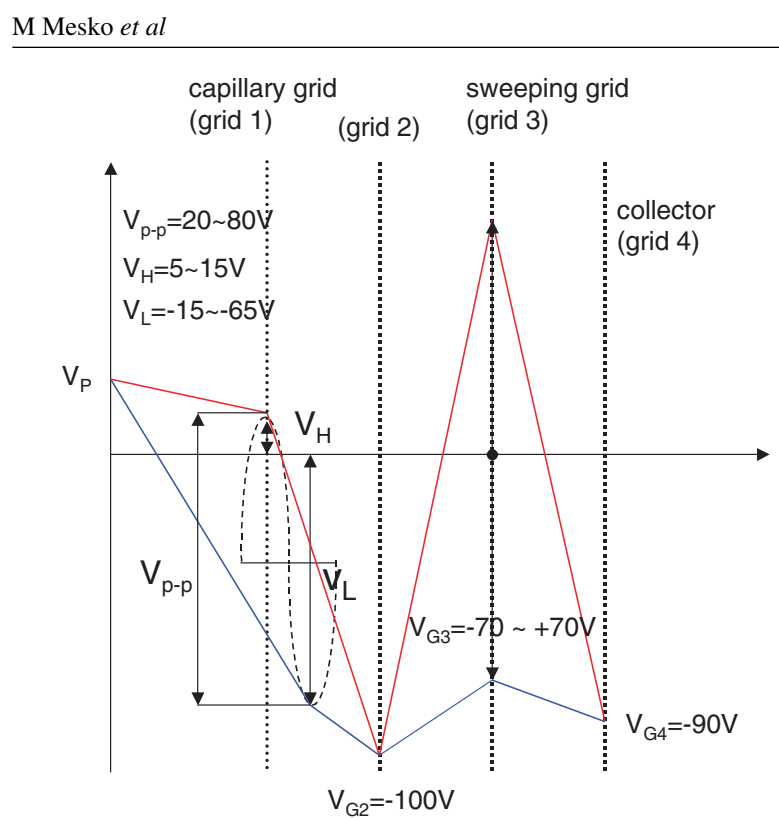

Figure 3. Potential distribution configuration of the IEA.

electron current, the potential of the second grid was set to $-100 \mathrm{~V}$, which is supposed to be sufficient to reject the incoming electrons. The analysing potential, $V_{\mathrm{g}}$, swept from $-70 \mathrm{~V}$ to $+70 \mathrm{~V}$, was applied on the third grid and the signal in the collector, $I_{\mathrm{c}}$, was measured as a function of the third grid potential, $V_{\mathrm{g}}$. Here, the collector was also biased to $-90 \mathrm{~V}$, so as to reflect any electrons, that may have passed through to the collector or were created in the analyser. The potential distribution configuration of the IEA is shown in figure 3 . The support for the substrate holder was not grounded, i.e. it was at floating potential.

A Langmuir probe made of tungsten wire with length $1 \mathrm{~mm}$ and a diameter of $0.5 \mathrm{~mm}$ was used to measure the plasma parameters such as electron temperature, plasma potential and plasma density. The probe was movable in the axial $(z)$ and radial $(r)$ directions in order to measure the spatial profiles of the plasma parameters.

The chamber was pumped out by the diffusion pump to pressures below $10^{-5}$ Torr, and during the operation, the pump was throttled and the pressure was adjusted by argon gas flow.

For optimal performance, the plasma was ignited by microwave power of $800 \mathrm{~W}$ at a pressure of $250 \mathrm{mTorr}$, where its production was characterized by the light layer pattern of the plasma with constant thickness near the fused silica plate. After this, the microwave power was decreased to $400 \mathrm{~W}$ and the gas pressure was continuously decreased down to the operating pressure of $10 \mathrm{mTorr}$, at which the plasma diffused to a large volume.

\section{Measurements and analysis of IEDFs}

Here, we describe the procedure for obtaining the IEDFs. The collector current, $I_{\mathrm{c}}$, is measured as a function of the retarding grid potential, $V_{\mathrm{g}}$, applied to the analysing grid, as shown schematically in figure 2 . The ions with enough energy, $E$, to overcome the electric field of the analysing grid, $E>e V_{\mathrm{g}}$, are recorded as a collector current, $I_{\mathrm{c}}$. The ions from the bulk of the plasma experience a kinetic energy gain when

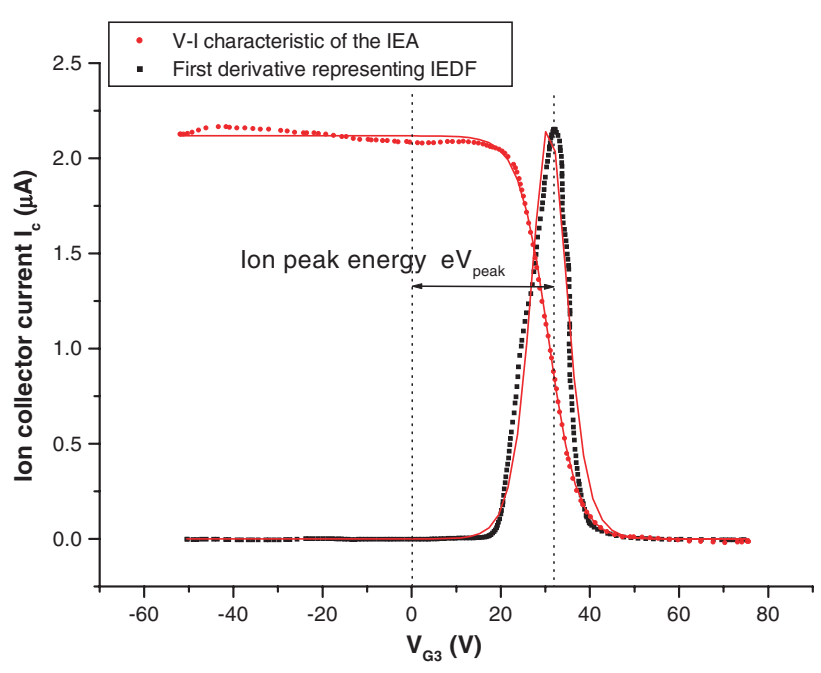

Figure 4. IEA characteristics: argon, 10 mTorr, power $400 \mathrm{~W}$, rf-bias voltage $V_{\mathrm{p}-\mathrm{p}}=80 \mathrm{~V}$, rf-bias frequency $f_{\mathrm{rf}}=13.56 \mathrm{MHz}$.

they fall through the sheath to the potential of the rf-biased substrate. In the absence of collisions, the ions will enter the first grid with average velocity $v=\left[2 e\left(V_{\mathrm{p}}-V_{\mathrm{s}}\right) / M\right]^{1 / 2}$, where $V_{\mathrm{p}}$ is the plasma potential and $V_{\mathrm{s}}$ is the potential of the first grid, i.e. on the substrate. The current, $I_{\mathrm{c}}$, on the collector is equal to the ion flux passing the analysing grid. Assuming that ions pass the capillary plate with velocities almost perpendicular to the substrate (due to the capillary plate angular resolution), the distribution function is assumed to be reduced to a one-dimensional distribution function, $F(V)$. The current collected in the one-dimensional case will be given by

$$
I_{\mathrm{c}}=n_{\mathrm{i}} e A_{\mathrm{c}}\left(\frac{k T_{\mathrm{i}}}{2 \pi M}\right)^{1 / 2} \exp \left[-\frac{M\left(v_{\text {min }}-v_{\text {peak }}\right)^{2}}{2 k T_{\mathrm{i}}}\right],
$$

where $n_{\mathrm{i}}$ is the ion density, $A_{\mathrm{c}}$ the collecting area, $M$ the mass of the ion, $T_{\mathrm{i}}$ the ion temperature, $k$ the Boltzmann constant, $v_{\text {min }}$ the minimum velocity necessary to overcome the analysing barrier, $M v_{\text {min }}^{2} / 2=e V_{\mathrm{g}}$ and $v_{\text {peak }}$ the most probable ion beam velocity.

In this case, the ion distribution function is proportional to the derivative of the measured ion current, $I_{\mathrm{c}}$, with respect to the analysing grid potential, $V_{\mathrm{g}},[15,16]$

$\frac{\mathrm{d} I_{\mathrm{c}}}{\mathrm{d} V_{\mathrm{g}}}=-n_{\mathrm{i}} e^{2} A_{\mathrm{c}}\left(\frac{1}{2 \pi M k T_{\mathrm{i}}}\right)^{1 / 2} \exp \left[-\frac{e\left(V_{\mathrm{g}}-V_{\text {peak }}\right)}{k T_{\mathrm{i}}}\right]$,

where $e$ is the elementary charge, $k$ the Boltzmann constant, $T_{\mathrm{i}}$ the ion temperature and $V_{\text {peak }}$ is the grid potential at which the peak in the ion distribution function is detected.

\section{Experimental results}

\subsection{Plasma parameters}

Plasma parameters adjacent to the substrate determine much of the plasma sheath. The plasma-dependent quantities such as the plasma potential, $V_{\mathrm{p}}$, plasma density, $n$, and electron temperature, $T_{\mathrm{e}}$, were determined from the Langmuir probe $V-I$ characteristic measured $5 \mathrm{~mm}$ above the substrate. 


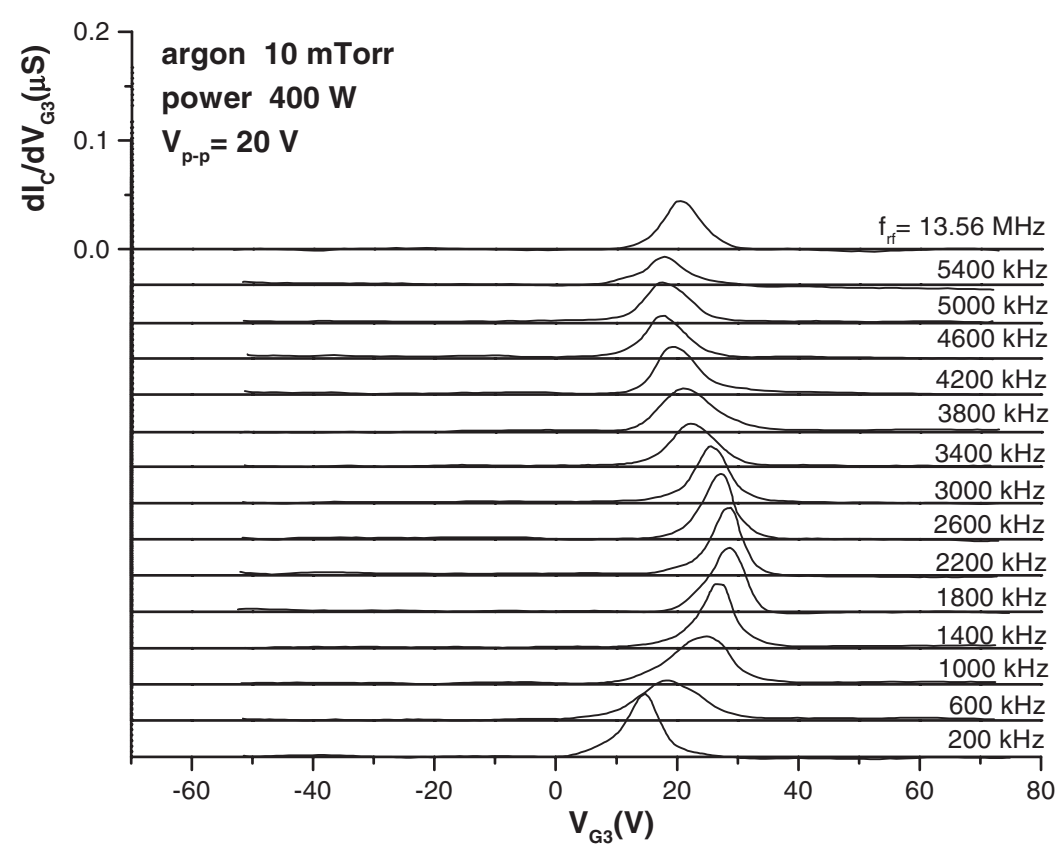

Figure 5. IEDFs measured at varying rf-bias frequencies, $V_{\mathrm{p}-\mathrm{p}}=20 \mathrm{~V}$, for argon plasma at pressure $10 \mathrm{mTorr}$, microwave power $400 \mathrm{~W}$.

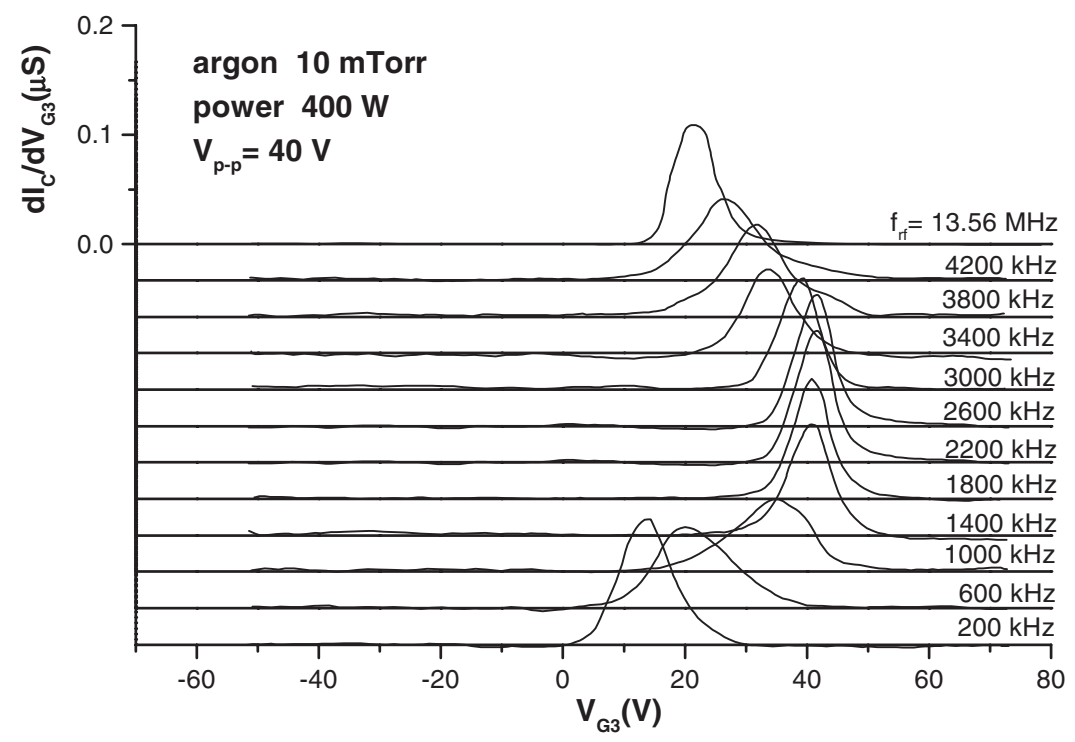

Figure 6. IEDFs measured at varying rf-bias frequencies, $V_{\mathrm{p}-\mathrm{p}}=40 \mathrm{~V}$, for argon plasma at pressure $10 \mathrm{mTorr}$, microwave power $400 \mathrm{~W}$.

The plasma parameters for the argon plasma at pressure $10 \mathrm{mTorr}$ and microwave power $400 \mathrm{~W}$ at a position $5 \mathrm{~mm}$ above the substrate are the following: electron temperature $T_{\mathrm{e}}=1.5 \mathrm{eV}$, plasma potential $V_{\mathrm{p}}=15 \mathrm{~V}$, plasma density $n=4 \times 10^{9} \mathrm{~cm}^{-3}$. This results in an ion plasma frequency, $f_{\mathrm{pi}}$, of $2100 \mathrm{kHz}$, where $f_{\mathrm{pi}}=\left(n e^{2} / 4 \pi^{2} \varepsilon_{0} M\right)^{1 / 2}$.

\subsection{IEDFs}

In this section, we show the results concerning the energies of ions incident on the substrate and show how it is possible to control incident ion energies.

In figure 4, typical curves obtained by our ion analyser are presented. However, using equations (1) and (2) we are not able to describe the measured $V-I$ characteristic and the first derivate representing the IEDF. We empirically found that the functions that can describe our measured data are the following:

$I_{\mathrm{c}}=n_{\mathrm{i}} e A_{\mathrm{c}}\left(\frac{k T_{\mathrm{i}}}{2 \pi M}\right)^{1 / 2}\left\{1+\exp \left[+\frac{e\left(V_{\mathrm{g}}-V_{\text {peak }}\right)}{k T_{\mathrm{i}}}\right]\right\}^{-1}$,

characterizing the collector current of the IEA, and the corresponding derived first derivative is

$$
\begin{aligned}
\frac{\mathrm{d} I_{\mathrm{c}}}{\mathrm{d} V_{\mathrm{g}}} & =-\frac{n_{\mathrm{i}} e^{2} A_{\mathrm{c}}}{\sqrt{2 \pi k M T_{\mathrm{i}}}}\left\{1+\exp \left[+\frac{e\left(V_{\mathrm{g}}-V_{\text {peak }}\right)}{k T_{\mathrm{i}}}\right]\right\}^{-2} \\
& \times \exp \left[+\frac{e\left(V_{\mathrm{g}}-V_{\text {peak }}\right)}{k T_{\mathrm{i}}}\right] .
\end{aligned}
$$




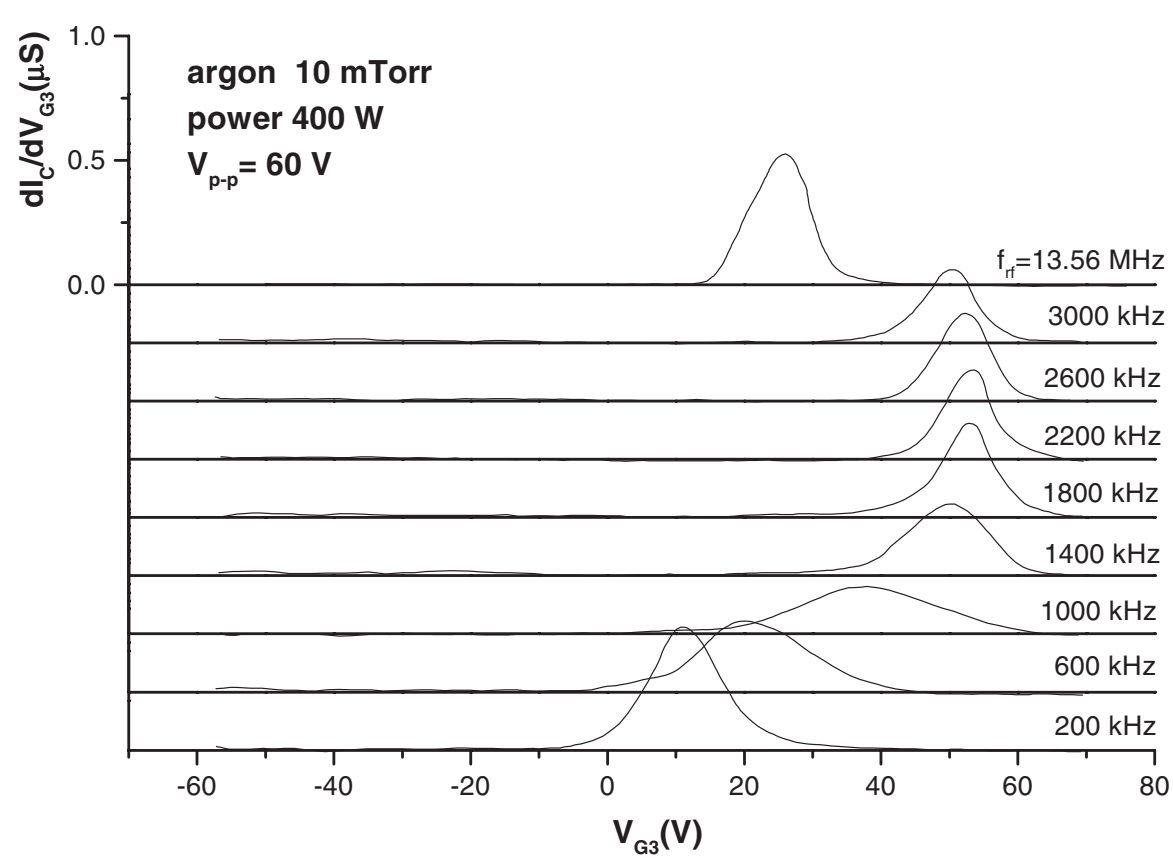

Figure 7. IEDFs measured at varying rf-bias frequencies, $V_{\mathrm{p}-\mathrm{p}}=60 \mathrm{~V}$, for argon plasma at pressure $10 \mathrm{mTorr}$, microwave power $400 \mathrm{~W}$.

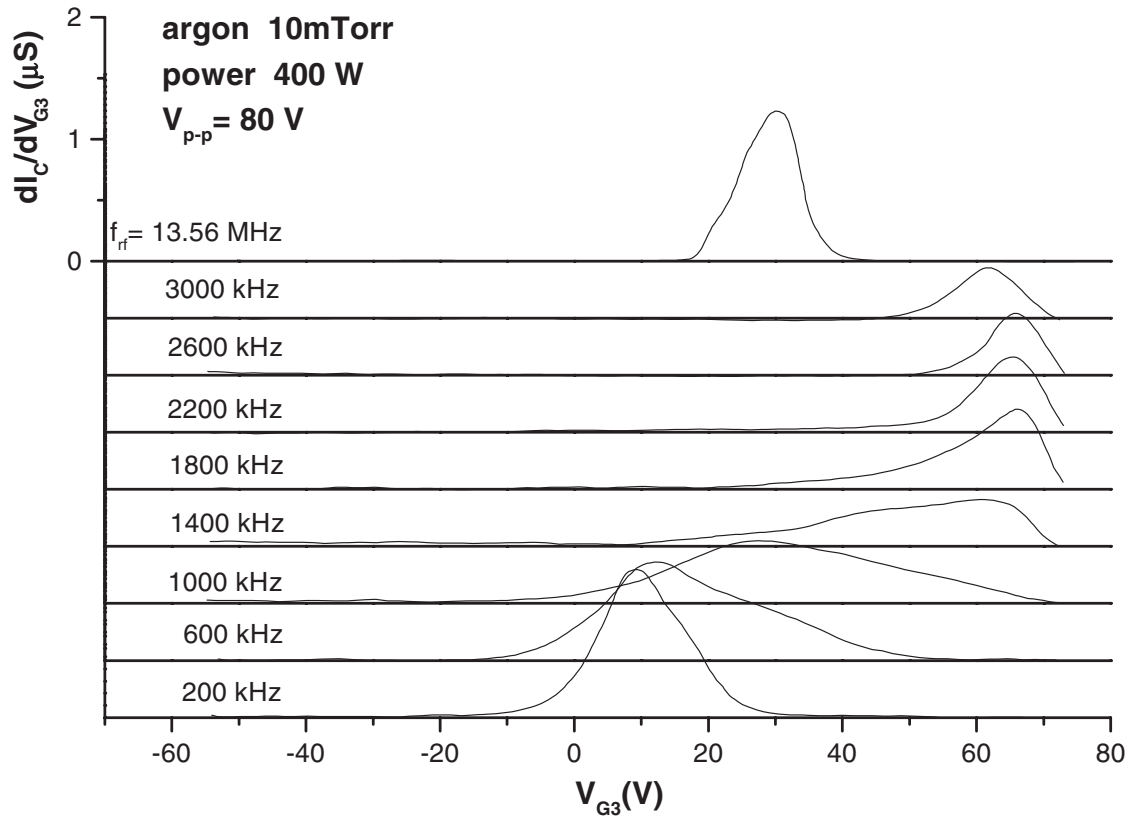

Figure 8. IEDFs measured at varying rf-bias frequencies, $V_{\mathrm{p}-\mathrm{p}}=80 \mathrm{~V}$, for argon plasma at pressure $10 \mathrm{mTorr}$, microwave power $400 \mathrm{~W}$.

Examples of our measured data fitted by functions describing by equations (3) and (4) are also shown in figure 4.

Figures 5-8 show the IEDF measured at various rf-bias frequencies, $f_{\text {rf }}$, with rf-bias peak-to-peak voltage $V_{\mathrm{p}-\mathrm{p}}=20$, 40, 60 and $80 \mathrm{~V}$. When the rf-bias frequency increases, the position of the ion peak, $V_{\text {peak }}$ (corresponding to the ion peak energy, $e V_{\text {peak }}$ ), shifts to the higher energies and approaches the rf-bias voltage, $V_{\mathrm{p}-\mathrm{p}}$, at around $f_{\mathrm{rf}} \approx 2200 \mathrm{kHz}$. This value corresponds well to the value of ion plasma frequency $f_{\mathrm{pi}} \approx 2100 \mathrm{kHz}$, calculated from the ion density measurements $5 \mathrm{~mm}$ above the substrate. For $f_{\mathrm{rf}}<f_{\mathrm{pi}}$, the IEDFs are similar to those of dc-bias, while for $f_{\mathrm{rf}}>f_{\mathrm{pi}}$ the position of the ion peak, $V_{\text {peak }}$, shifts to lower energies as the ions cannot sufficiently follow the oscillating rf-bias field.

Figure 9 shows the IEDF for rf-bias $V_{\mathrm{p}-\mathrm{p}}=20-80 \mathrm{~V}$, at rf-bias frequency $f_{\mathrm{rf}}=200 \mathrm{kHz}$, as an example of the data in the region $f_{\mathrm{rf}} \ll f_{\mathrm{pi}}$. It is seen that the ion peak position, $V_{\text {peak }}$, does not change with increasing bias voltage, $V_{\mathrm{p}-\mathrm{p}}$, while the ion peak signal intensity increases with increasing rf-bias, $V_{\mathrm{p}-\mathrm{p}}$. This situation $\left(f_{\mathrm{rf}} \ll f_{\mathrm{pi}}\right)$ is similar to that of the dc-bias of the substrate, where no change of peak position, $V_{\text {peak }}$, was observed [17].

Figure 10 shows the IEDF for dc-bias $V_{\mathrm{p}-\mathrm{p}}=20-80 \mathrm{~V}$, at rf-bias frequency $f_{\text {rf }}=2200 \mathrm{kHz} \approx f_{\text {pi }}$. It is seen that the 


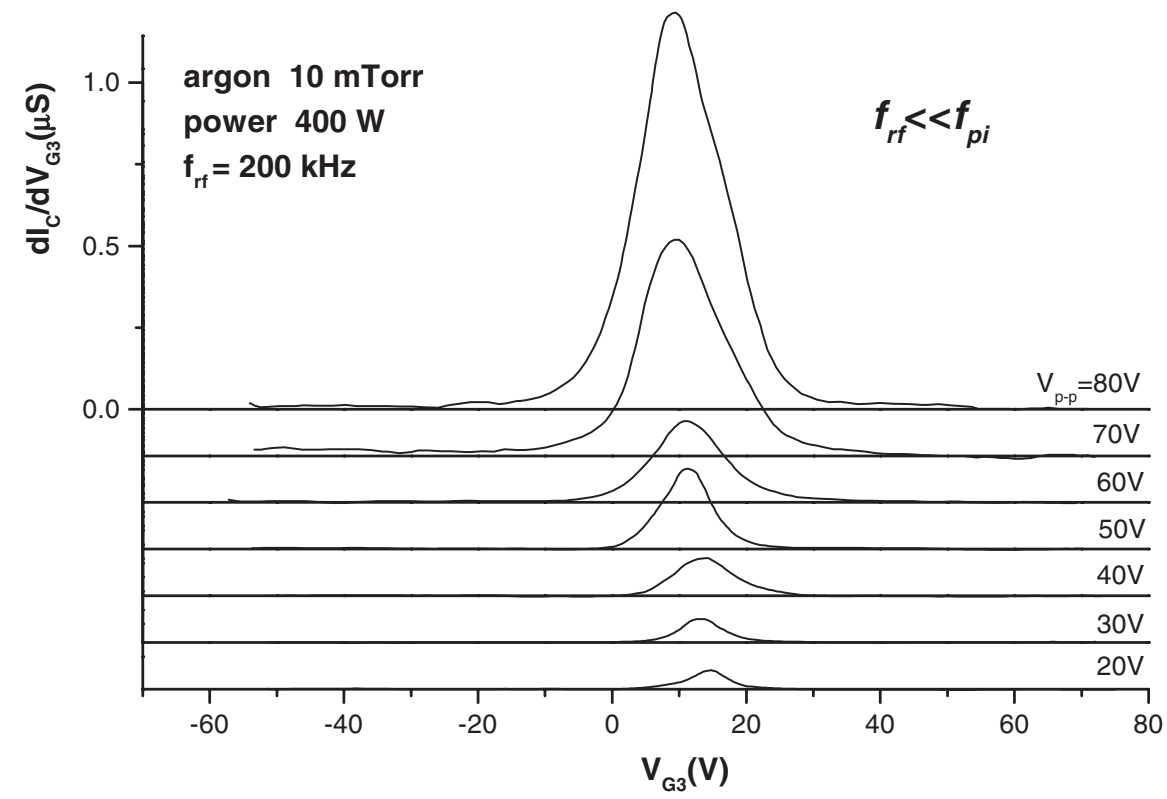

Figure 9. IEDFs measured with rf-bias applied at $200 \mathrm{kHz}$ at varying rf-bias voltages.

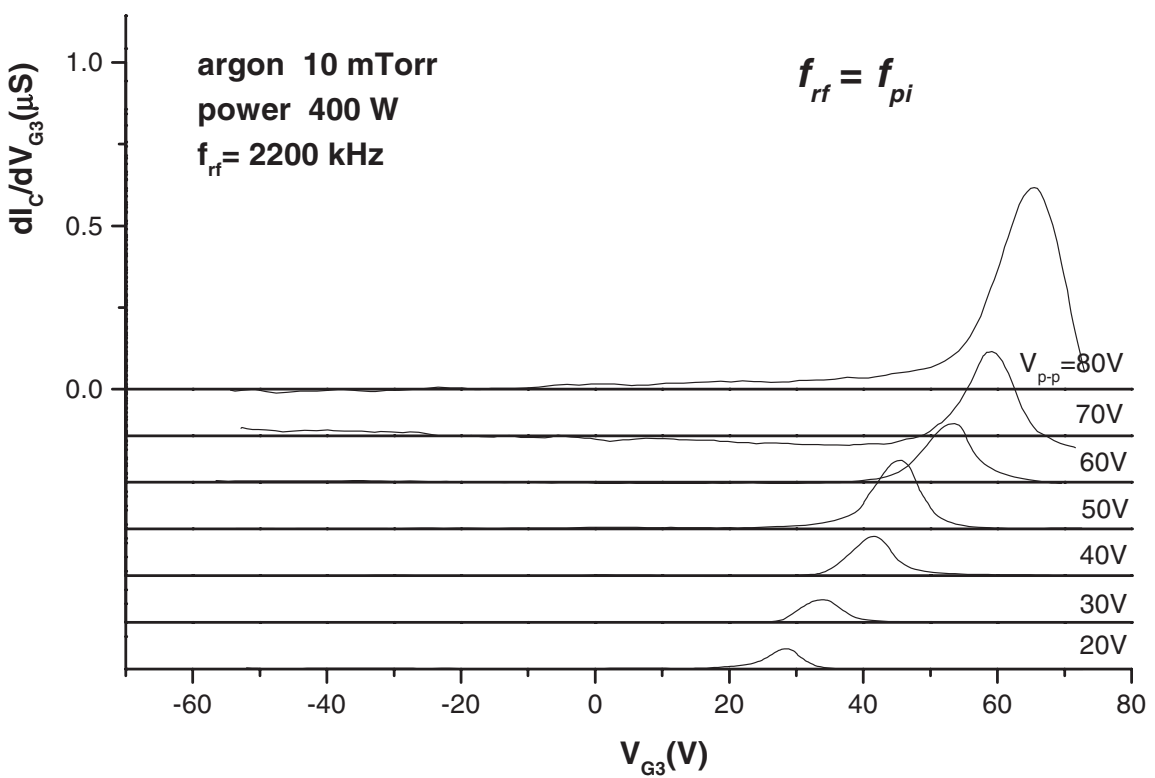

Figure 10. IEDFs measured with rf-bias applied at $2200 \mathrm{kHz}$ at varying rf-bias voltages.

ion signal intensity increases with increasing rf-bias, $V_{\mathrm{p}-\mathrm{p}}$, and also that the ion peak position, $V_{\text {peak }}$, increases with increasing rf-bias $V_{\mathrm{p}-\mathrm{p}}$. In fact, the ion peak position, $V_{\text {peak }}$, changed from 25 to $65 \mathrm{~V}$ when the rf-bias, $V_{\mathrm{p}-\mathrm{p}}$, was changed from 20 to $80 \mathrm{~V}$. We can see the position of the ion peak, $V_{\text {peak }}$, is nearly proportional to the rf-bias voltage, $V_{\mathrm{p}-\mathrm{p}}$. As all this happens at $f_{\mathrm{rf}} \approx f_{\mathrm{pi}}$, some characteristics of resonance behaviour can be expected. In this case, it is exhibited by the proportionality of the peak position, $V_{\text {peak }}$, with the applied rf-bias voltage, $V_{\mathrm{p}-\mathrm{p}}$, while this proportionality is not detected for frequencies lower (figure 9) or higher (figure 11, discussed later) than $f_{\mathrm{pi}}$. This might suggest an explanation for the oscillation mechanism by Chen [18].

Figure 11 shows the IEDF for rf-bias $V_{\mathrm{p}-\mathrm{p}}=20-80 \mathrm{~V}$, at rf-bias frequency $f_{\mathrm{rf}}=13.56 \mathrm{MHz}$; for the case when $f_{\text {rf }} \gg f_{\text {pi }}$, it can be seen that the signal intensity increases with increasing rf-bias voltage, $V_{\mathrm{p}-\mathrm{p}}$. The ion peak position, $V_{\text {peak }}$, is shifted from 15 to $30 \mathrm{~V}$ as the rf-bias voltage is changed from 20 to $80 \mathrm{~V}$. As in this case, we are working at much higher frequencies, the ions are no longer able to follow the rf field, and the measured data start to differ from the case of resonance, $f_{\mathrm{rf}} \approx f_{\mathrm{pi}}$; however, this is much more complicated behaviour and should be studied in more detail. One approach to this theme has been made by investigation of the ion collection using a high frequency probe [19].

\section{Conclusions}

In this work, we have successfully used a differentially pumped IEA with low angular resolution to measure IEDFs as functions 


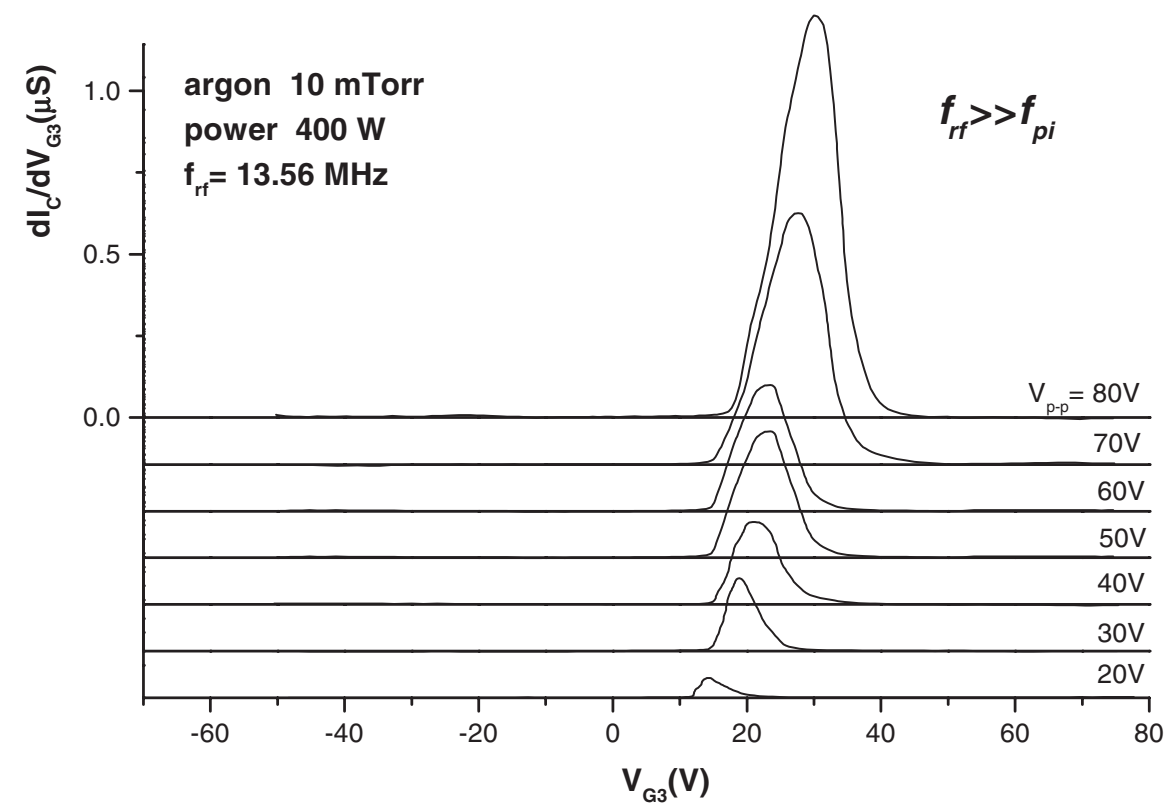

Figure 11. IEDFs measured with rf-bias applied at $13.56 \mathrm{MHz}$ at varying rf-bias voltages.

of the rf-bias voltage and the rf-bias frequency applied to the substrate. We found that (i) at low frequency rf-bias, $f_{\mathrm{rf}} \approx 0.1 f_{\mathrm{pi}}$, the ion peak energy is independent of the rf-bias voltage and remains at a position around the plasma potential, (ii) at a rf-bias frequency approximately equal to the ion plasma frequency, $f_{\mathrm{rf}} \approx f_{\mathrm{rf}}$, the ion peak energy is proportional to the rf-bias voltage and (iii) at a high frequency rf-bias, $f_{\mathrm{rf}} \approx 10 f_{\mathrm{pi}}$, the ion peak energy slightly increases with rf-bias voltage. Control of rf-bias frequency and rf-bias voltage can be used for fine adjustment of the energy of ions impinging upon an exposed surface.

\section{Acknowledgments}

Two of the authors, M Mesko and P Cicman, were sponsored by a Japanese Government (Monbusho) Scholarship for Special Joint Program for Foreign Postgraduate Students.

\section{References}

[1] Hopwood J 1993 Appl. Phys. Lett. 62940

[2] Kortshagen U and Zethoff M 1995 Plasma Sources Sci. Technol. 4541
[3] Matsoka M and Ono O 1987 J. Vac. Sci. Technol. A 625

[4] Holber W M and Foster J 1990 J. Vac. Sci. Technol. A 83720

[5] Gibson G W Jr, Sawin H H, Tepermeister I, Ibbotson D E and Lee J T C 1994 J. Vac. Sci. Technol. B 122333

[6] Charles C, Boswell R W and Porteous R K 1992 J. Vac. Sci. Technol. A 398

[7] Ohtsu Y, Tochitani G, Fujita H, Zhang J, Setsuhara Y and Miyake S 1997 Japan. J. Appl. Phys. 36 (part 1) 4620

[8] Charles C 1993 J. Vac. Sci. Technol. A 11157

[9] Kim J H and Chang H Y 1996 Phys. Plasmas 31462

[10] Martin D and Oechsner H 1996 Vacuum 471017

[11] Aanesland A and Fredriksen A 2001 J. Vac. Sci Technol. A 19 2446

[12] Benck E C, Schwabedissen A, Gates A and Roberts J R 1998 J. Vac. Sci Technol. B 16306

[13] Stenzel R L, Williams R, Aguero R, Kitazaki K, Ling A, McDonald T and Spitzer J 1982 Rev. Sci. Instrum. 531027

[14] Ikushima T, Tochitani G and Fujita H 1995 J. Phys. D: Appl. Phys. 281851

[15] Okuno Y, Ohtsu Y and Fujita H 1993 J. Appl. Phys. 745990

[16] Sugawara M 1998 Plasma Etching: Fundamentals and Applications (Oxford: Oxford University Press) chapter 4

[17] Ohtsu Y, Mori K and Fujita H 1999 Jpn. J. Appl. Phys. 38 4393-6

[18] Chen F F 1990 Introduction to Plasma Physics and Controlled Fusion (New York: Plenum) chapter 4

[19] Chen F F, Huddestone R H and Leonard S L 1965 Plasma Diagnostic Techniques (New York: Acadamic) chapter 4 